\title{
circKL inhibits the growth and metastasis of kidney cancer by sponging miR-182-5p and upregulating FBXW7
}

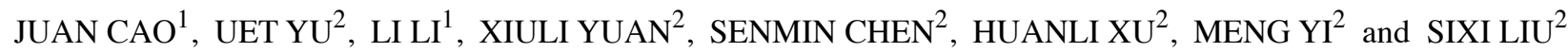 \\ Departments of ${ }^{1}$ Pathology and ${ }^{2}$ Hematology and Oncology, \\ Shenzhen Children's Hospital, Shenzhen, Guangdong 518038, P.R. China
}

Received August 1, 2021; Accepted January 19, 2022

DOI: $10.3892 /$ or.2022.8286

\begin{abstract}
Circular RNAs (circRNAs) are a type of non-coding RNA with important roles in the regulation of various biological processes involved in malignant progression. However, the potential molecular mechanisms and roles of circRNAs in kidney cancer have remained to be fully elucidated. In a previous study by our group, high-throughput microarray sequencing data were analyzed to determine the differentially expressed circRNAs in kidney cancer. In this analysis, a novel circRNA (hsa_circ_0100312, named circKL) was identified as a frequently downregulated circRNA in kidney cancer cells and tissues by reverse transcription-quantitative PCR. In the present study, Cell Counting Kit-8, colony formation, Transwell, wound-healing and mouse xenograft assays as well as a lung metastasis experiment were performed to confirm the functions of circKL. The experiments confirmed that circKL overexpression significantly inhibited the proliferation, migration, tumor growth and metastasis of kidney cancer both in vitro and in vivo. The potential molecular mechanisms of circKL were investigated by performing dual-luciferase and RNA immunoprecipitation assays. Western blot assays confirmed that overexpression of circKL significantly increased the protein level of F-box and WD repeat domain containing 7 (FBXW7). All results suggested that circKL suppressed the growth and migration of kidney cancer by sponging microRNA (miR)-182-5p and upregulating FBXW7 expression. Overall, the circKL/miR-182-5p/FBXW7 axis was indicated to have a key role in the growth and metastasis of kidney cancer and may be targeted as a novel therapeutic strategy.
\end{abstract}

\section{Introduction}

The incidence rates of kidney cancer have been increasing worldwide, which has only received small amounts of academic

Correspondence to: Dr Sixi Liu, Department of Hematology and Oncology, Shenzhen Children's Hospital, 7019 Yitian Road, Shenzhen, Guangdong 518038, P.R. China

E-mail: tiger647@126.com

Key words: hsa_circ_0100312, circular RNA, F-box and WD repeat domain containing 7, competing endogenous RNAs, kidney cancer attention. For instance, nephroblastoma (also known as Wilms' tumor), which is a type of kidney cancer, accounts for $\sim 7 \%$ of all pediatric malignancies and $90 \%$ of all pediatric renal tumors (1). The most pervasive and effective treatment for kidney cancer is nephrectomy combined with chemotherapy (2). Although $90 \%$ of pediatric patients with kidney cancer survive following systematic treatment (3), conventional treatment has proven to be ineffective in a subset of those with high-risk kidney cancer (4). The majority of such patients relapse several years after nephrectomy $(5,6)$. Therefore, it is necessary to elucidate advanced therapeutic strategies to improve the prognosis of patients with high-risk kidney cancer through an improved understanding of the molecular pathogenesis of the disease.

Circular RNAs (circRNAs) are a novel type of endogenous non-coding RNA (ncRNA) that are formed by covalently closed loops. circRNAs are single-stranded ncRNA transcripts that are present in cells. They bind to microRNAs (miRNAs or miRs) or other molecules through various molecular mechanisms (7). circRNAs abundantly exist in the eukaryotic transcriptome and consist of the precursor mRNA reverse splicing sequences of exons or introns without upstream heads or downstream tails (8). circRNAs have a circular structure and are resistant to RNA exonuclease, which makes them stable when compared with linear mRNA transcripts (9). In the intracellular microenvironment, circRNAs regulate the expression of important oncogenes through various integrated molecular mechanisms, including miRNA binding, protein interaction and novel small molecular peptide encoding (10). Innovative circRNA-sequencing technology and bioinformatic analysis have resulted in the increased study and characterization of circRNAs (11). circRNAs are regulators of various diseases, including diabetes, neurological diseases, immune diseases, heart failure and cancer (12). Among them, the circRNA for miRNA-7/circular RNA ciRS-7 [cerebellar degeneration related protein 1 antisense (CDRlas)] is the most well-studied circRNA. CDRlas is an oncogene that promotes the growth, migration, chemotherapeutic resistance and immunodeficiency of various types of tumors through sponging miR-7 (13-18). circRNA-002178 may act as a competing endogenous (ce)RNA to upregulate the expression of programmed death-ligand 1 and programmed cell death protein 1 in lung adenocarcinoma, which mediates the immune escape of the tumor (19). Circ0006916 is regulated by trinucleotide repeat-containing $6 \mathrm{~A}$ and has been determined to be a tumor promoter in lung cancer cells (20). 
circRNA polo-like kinase-1 and hsa_circ_0002453/circRAD18 are considered to be tumor-promoting circRNAs, as they reduce apoptosis and accelerate proliferation in triple-negative breast cancer (21-23). However, research on the potential molecular mechanisms and roles of circRNAs in kidney cancer remains insufficient.

In the present study, a frequently downregulated novel circRNA (hsa_circ_0100312, named circKL) in kidney cancer was identified by analyzing circRNA microarray profiling data of a previous study by our group. A series of experiments and bioinformatic analysis were performed to examine the functions and mechanisms of circKL in kidney cancer. The present study demonstrated the pivotal role of the circKL-miR-182-5p-F-box/WD repeat-containing protein 7 (FBXW7) axis in kidney cancer growth and metastasis though the mechanism of ceRNAs. Thus, circKL may have the potential to be a novel therapeutic target and biomarker for kidney cancer.

\section{Materials and methods}

Clinical samples, data and ethics approval. Kidney cancer (mainly referring to nephroblastoma) and corresponding non-cancerous kidney tissues were collected from 10 patients at Shenzhen Children's Hospital (Shenzhen, China) between December 2019 and June 2020. The distance between the tumor and the matched normal adjacent tissue was $>2 \mathrm{~cm}$ and it was histologically confirmed to be non-cancerous. The inclusion criteria were as follows: i) Diagnosed with kidney cancer; ii) had not received other adjuvant treatments including chemotherapy and radiotherapy prior to surgery; and iii) agreed to participate in the study. The exclusion criteria were as follows: i) Failed to cooperate with researchers; and ii) diagnosed with other diseases. These patients included 6 male and 4 female patients with an age range of 7-50 months (average age, $29.04 \pm 14.13$ months). The age below 18 years was the most frequently used cutoff point for kidney cancer in China (24). The present study was approved by the Ethics Committee of Shenzhen Children's Hospital (Shenzhen, China). Written informed consent was obtained from the parents or legal guardians of the patients with kidney cancer prior to study enrollment. Animal experiments were performed in accordance with the guiding principles of the Institutional Animal Care and Use Committee of Shenzhen Children's Hospital (Shenzhen, China). Detailed information regarding the demographic and clinicopathological characteristics is provided in Table SI.

Cell culture. A total of three kidney cancer cell lines were used in the present study: The kidney Ewing sarcoma cell line SKNEP1, the kidney rhabdoid tumor cell line G401 and the kidney nephroblastoma cell line HANB. A cell line originally derived from human embryonic kidney cells, 293T, was also used. All cell lines were purchased from the Type Culture Collection of the Chinese Academy of Sciences and were cultured in RPMI-1640 and McCoy's 5A medium (HyClone; Cytiva) supplemented with 10\% FBS (HyClone; Cytiva) at $37^{\circ} \mathrm{C}$ with $5 \% \mathrm{CO}_{2}$. The authenticity of all cell lines was verified by DNA fingerprinting (short tandem repeat profiling).

Vector construction and transfection. The full-length sequence of circKL (Geneseed Biotech Co., Ltd.) was cloned into a
pLCDH vector (BioVector NTCC, Inc.), which was subsequently cotransfected with two assistant vectors pMD2.G (cat. no. 12259; BioVector NTCC, Inc.) and psPAX2 (cat. no. 12260; BioVector NTCC, Inc.) into 293T cells to produce a lentivirus. The control group was treated with the lentiviral vector. Each cell line was then transfected with the circKL overexpression lentivirus. Cells were subsequently selected following exposure to puromycin for 7 days, after which the results were validated by reverse transcription-quantitative (RT-q)PCR analysis.

Total RNA extraction and RT-qPCR. According to the manufacturer's protocol, total RNA was extracted from kidney cancer tissues or cultured cell lines using the TRIzol ${ }^{\circledR}$ reagent (Invitrogen; Thermo Fisher Scientific, Inc.), after which cDNA was synthesized using the PrimeScript ${ }^{\circledR}$ RT Master Mix (Takara Bio, Inc.). NE-PER Nuclear and Cytoplasmic Extraction Reagents (cat. no. 78833; Thermo Scientific) were utilized to isolate the nuclear and cytoplasmic portions of cellular RNA. Subsequently, qPCR was performed using the $\mathrm{SYBR}^{\circledR}$ Premix Ex Taq ${ }^{\mathrm{TM}}$ II kit (Code: DRR081; Takara Bio, Inc.) and an ABI 7900 Sequence Detection system (Applied Biosystems; Thermo Fisher Scientific, Inc.) according to the manufacturer's protocol. The thermocycling conditions were as follows: $95^{\circ} \mathrm{C}$ for $1 \mathrm{~min}$, followed by 40 cycles of $95^{\circ} \mathrm{C}$ for $10 \mathrm{sec}$ and $60^{\circ} \mathrm{C}$ for $30 \mathrm{sec}$ according to the manufacturer's protocol. The primers used for qPCR were as follows: circKL forward, 5'-ATGGAA TCGATGACGGGCTG-3' and reverse, 5'-GCTTAGGGCAAT GGACACCT-3'; linear KL forward, 5'-GTGCGTCCATCTGGG ATACG-3' and reverse, 5'-TGTCGCGGAAGACGTTGTT-3'; circ0056949 forward, 5'-GCATCTTTGACTGCCCCAATG-3' and reverse, 5'-ATCCACACAGTCGTTGCGTT-3'; circ0056860 forward, 5'-ACGTGTTATTGATCTTCGCTGT-3' and reverse, 5'-TTCAGCATCATTTGTCAATGGC-3'; circ0056861 forward, 5'-TCTTCGCTGTAACCCAAGAACA-3' and reverse, 5'-GGA GACAGGGTTTTCGATGA-3'; circ0039504 forward, 5'-AGC TATGGCTGGAACTTCACC-3' and reverse, 5'-ACATTCCGA AGAAGGTGCCAT-3'; miR-182-5p forward, 5'-ATCACTTTT GGCAATGGTAGAACT-3' and reverse, 5'-TATGGTTTTGAC GACTGTGTGAT-3'; GAPDH forward, 5'-ACAACTTTGGTA TCGTGGAAGG-3' and reverse, 5'-GCCATCACGCCACAG TTTC-3'. The $2^{-\triangle \triangle C q}$ method was used to determine the fold change of expression (25).

Western blot analysis. Total protein was isolated from kidney tissues and cells using radioimmunoprecipitation (RIP) assay lysis buffer (Nanjing KeyGen Biotech Co., Ltd.), which was then added to PMSF to prevent degradation. Equal quantities of protein $(20 \mu \mathrm{g})$ were resolved by $10 \%$ SDS-PAGE, separated and transferred to PVDF membranes (EMD Millipore) for $2 \mathrm{~h}$ at $300 \mathrm{~mA}$. After blocking the membranes with $5 \%$ skimmed milk (Nestle) for $2 \mathrm{~h}$ at room temperature, the membrane was then incubated with the following antibodies overnight at $4^{\circ} \mathrm{C}$ : Anti-FBXW7 (1:1,000 dilution; cat. no. ab109617; Abcam) and anti-GAPDH (1:1,000 dilution; cat. no. ab8245; Abcam). Samples were then incubated with horseradish-peroxidase-conjugated secondary antibody (1:2,000 dilution; cat. no. ab288151; Abcam) at room temperature for $1 \mathrm{~h}$. GAPDH was used as an internal control. Band densitometry analysis was performed using ImageJ software (version 1.8.0.112; National Institutes of Health). 
RNase R digestion assay. After $3 \mu \mathrm{g}$ of total RNA was extracted from SKNEP1 kidney cancer cells, samples were treated with Ribonuclease R (RNase R) (5 U/ $\mu$ g; cat. no. R0301; Geneseed, Inc.) or control solution for $20 \mathrm{~min}$ at $37^{\circ} \mathrm{C}$. Purification was then performed using an RNeasy MinElute Cleanup Kit (cat. no. 74204; Qiagen $\mathrm{GmbH}$ ) and the RNAs were quantified by RT-qPCR analysis.

Actinomycin D assay. SKNEP1 kidney cancer cells were exposed to $3 \mu \mathrm{g} / \mathrm{ml}$ actinomycin D (cat. no. SBR00013; MilliporeSigma) to degrade the linear mRNA transcript for $0,8,16$ or $24 \mathrm{~h}$. SKNEP1 cells were subsequently harvested, after which the stability of circKL and linear KL mRNA was analyzed by RT-qPCR.

Cell Counting Kit-8 (CCK-8) assay. G401 and SKNEP1 kidney cancer cells were digested and resuspended. Empty vector-transfected and circKL overexpression vector-transfected cancer cells (each, 5,000 cells/well) were seeded into a 96 -well plate and incubated for $48 \mathrm{~h}$ at $37^{\circ} \mathrm{C}$. Subsequently, $10 \mu \mathrm{l}$ CCK-8 solution (cat. no. C0037; Beyotime Institute of Biotechnology) was added, followed by incubation at $37^{\circ} \mathrm{C}$ for $1 \mathrm{~h}$ prior to optical density measurement at $450 \mathrm{~nm}$ using a microtiter plate reader (Epoch 2; BioTek Instruments, Inc.).

Colony-formation assay. To assess the colony formation ability of the cells $1 \times 10^{3}$ cells were seeded in six-well plates and incubated at $37^{\circ} \mathrm{C}$ for 2 weeks. When macroscopic colonies (>50 cells) were evidently observed, the cells were fixed with $100 \%$ methanol for $15 \mathrm{~min}$ at room temperature and stained with $0.5 \%$ crystal violet for $10 \mathrm{~min}$ at room temperature. The colonies were counted using an inverted light microscope (magnification, x100; Carl Zeiss AG). The numbers of colonies were then counted and measured using ImageJ software (version 1.8.0.112; National Institutes of Health). The colony formation efficiency was calculated as the number of colonies/plated cells $\times 100 \%$.

Transwell assay. A total of $3 \times 10^{4}$ cells in serum-free RPMI-1640 medium (HyClone; Cytiva) were resuspended and added to the upper chamber of Transwell plates $(8 \mu \mathrm{m}$ pore size; Cell Biolabs, Inc.). The upper chamber of Transwell plates were not coated with Matrigel ${ }^{\circledR}$ for migration assays. Furthermore, $600 \mu \mathrm{l}$ medium containing $10 \%$ FBS was added to the lower chamber. After incubation for $24 \mathrm{~h}$ at $37^{\circ} \mathrm{C}$, cells on the upper side of the filter were removed using a cotton swab. The cells which had migrated to the lower surface of the membrane were fixed using $4 \%$ paraformaldehyde and stained using $0.5 \%$ crystal violet at $37^{\circ} \mathrm{C}$ for $15 \mathrm{~min}$. Cells were then counted in five different fields (magnification, x200) under a light microscope (Carl Zeiss AG).

Wound-healing assay. G401 and SKNEP1 kidney cancer cells $\left(4 \times 10^{5}\right)$ were seeded in 6-well plates and transfected with vector or circKL. Subsequently, after a 70-80\% confluent culture was reached, a linear wound was scratched with a sterile $200-\mu 1$ pipette tip. Cells were washed 3 times with PBS and cultured with serum-free medium, after which cell wounds were imaged using an inverted microscope (Carl Zeiss AG) at 0 and $24 \mathrm{~h}$. The degree of wound healing was expressed as the change in width between the two time-points. Representative images were obtained using a light microscope (magnification, x100;
Carl Zeiss AG) and analyzed using ImageJ software (version 1.8.0.112; National Institutes of Health).

Dual-luciferase reporter assay. G401 or SKNEP1 kidney cancer cells were seeded into 96-well-plates ( $5 \times 10^{3}$ cells/well). miR-182 mimics (5'-UUUGGCAAUGGUAGAACUCACACU-3') and negative control mimics (miR-NC) (5'-UCACAACCUCCU AGAAAGAGUAGA-3') were obtained from Guangzhou RiboBio Co., Ltd. The complementary DNA fragment of G401 or SKNEP1 kidney cancer cells containing the wild-type (WT) or mutant type (MUT) sequence from the targeted 3'-untranslated region (UTR) of FBXW7 were subcloned downstream of the luciferase gene using the psiCHEK-2 vector (cat. no. C8021; Promega Corporation). Sequences of primers used to amplify the targeted 3'-UTR of FBXW7 mRNA were as follows: Forward, 5'-CCACTGACAGCTAGACACCTA-3' and reverse, 5'-GAACCCAGGACAACTTGCCA-3'. The plasmid with the MUT sequence from the 3'-UTR of FBXW7 mRNA was generated using a Site-Directed Mutagenesis Kit (Shanghai Yeasen Biotechnology Co., Ltd.). With this kit, the binding sites predicted by TargetScan (http://www.targetscan.org) of miR-182-5p and circKL in the 3'-UTR of FBXW7 mRNA were mutated. For transfection, cells were seeded into 24-well plates and cultured overnight. miR-182 mimics (10 $\mu \mathrm{l})$ or miR-NC (10 $\mu \mathrm{l})$ and luciferase reporter plasmid containing the WT or MUT 3'-UTR of FBXW7 (5 $\mu \mathrm{g})$ were transfected into cells using Lipofectamine ${ }^{\circledR} 3000$ (Thermo Fisher Scientific, Inc.) reagent $48 \mathrm{~h}$ prior to performing dual-luciferase reporter assays. Firefly and Renilla luciferase activities were examined by employing a Dual-Luciferase Reporter Assay system (Promega Corporation) in accordance with the manufacturer's protocol. Renilla luciferase activity was used as a normalization control.

RIP. RIP assays for argonaute RISC catalytic component 2 (AGO2) protein were performed using an anti-AGO2 antibody (EMD Millipore) according to the manufacturer's protocols. The relative expression levels of circKL, FBXW7 and miR-182-5p were assessed after RNA purification. For the MS2-based (Escherichia coli Bacteriophage MS2-based) immunoprecipitation assays, MS2 binding site Renilla luciferase (MS2bs-Rluc), MS2bs-circKL and MS2bs-circKL-mutant type (mut) plasmids were constructed using a pcDNA3.1 vector (cat. no. V79020; Invitrogen; Thermo Fisher Scientific, Inc.). Subsequently, $5 \mu \mathrm{g}$ MS2bs-Rluc, MS2bs-circKL or MS2bs-circKL-mt was transfected into G401 and SKNEP1 cells using Lipofectamine ${ }^{\circledR} 3000$ reagent (Invitrogen; Thermo Fisher Scientific, Inc.) prior to performing immunoprecipitation. Normal Mouse immunoglobulin G (IgG) (dilution, 1:150; cat. no. 17-700; EMD Millipore) served as the control control. The degree of miR-182-5p enrichment was measured by RT-qPCR after purification of RNA complexes. For the in vitro assays, three replicates were performed for each experiment.

Mouse xenograft study. SKNEP1 kidney cancer cells $\left(2 \times 10^{7}\right)$ that stably overexpressed circKL, or control vectors for the control group, were subcutaneously injected into randomly allocated BALB/c nude mice (five mice per circKL group and vector group; body weight, 20-25 g; age, 4 weeks). A total of $10 \mathrm{BALB} / \mathrm{c}$ nude mice were purchased from Shanghai Laboratory Animal Research Center. All mice were housed 

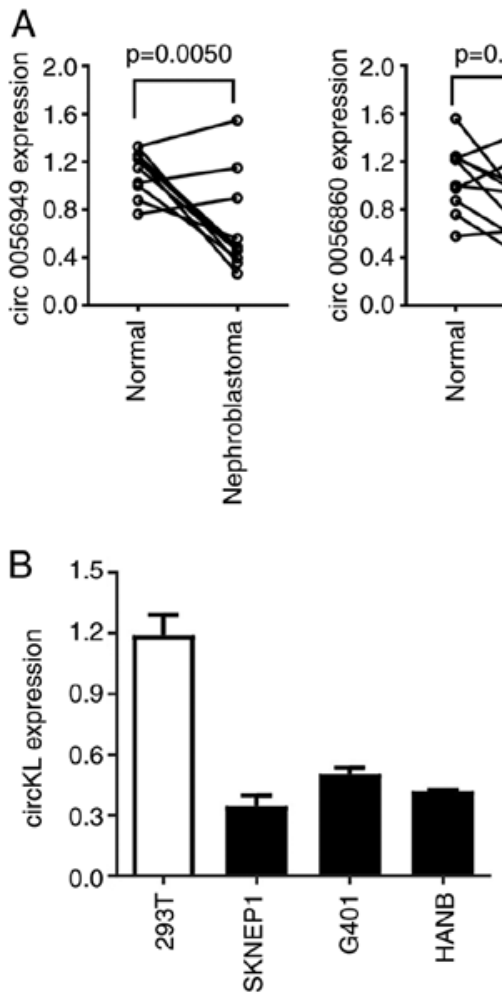
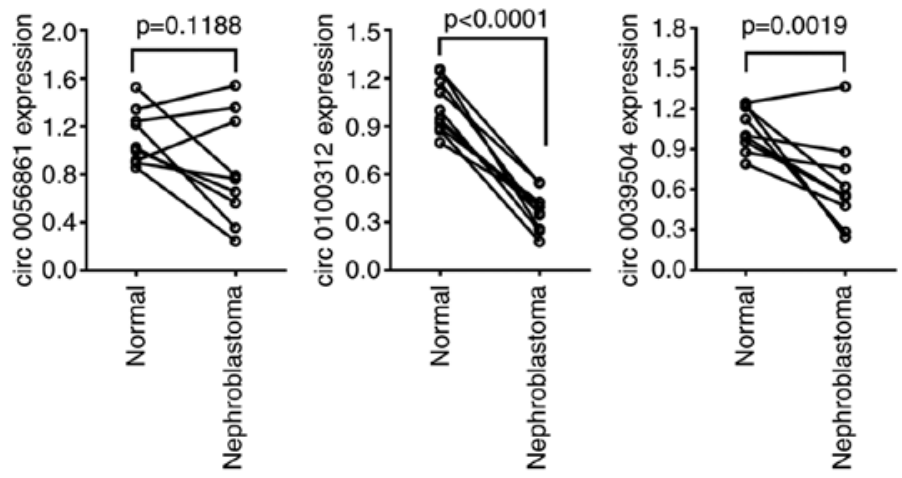

$\mathrm{c}$

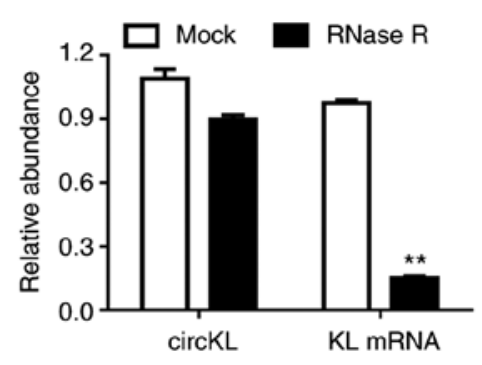

D

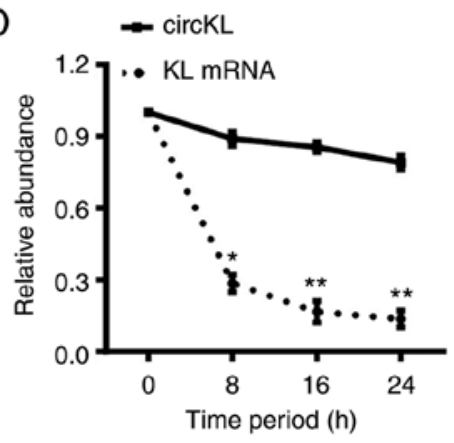

Figure 1. circKL is downregulated in kidney cancer. (A) Relative expression levels of the top five downregulated circRNAs between kidney cancer tissues (n=10) and adjacent normal kidney tissues $(n=10)$ were detected by reverse transcription-quantitative PCR analysis. (B) Relative expression levels of circKL in the normal 293T cell line and kidney cancer cell lines. (C) The circular features of circKL were tested by an RNase R assay in the SKNEP1 cell line. (D) Circular transcripts of circKL were more stable than its linear KL mRNA transcripts as determined by an actinomycin D treatment assay in the SKNEP1 cell line. The cell assays were performed as three biological replicates. ${ }^{* *} \mathrm{P}<0.01$ vs. mock group or the normal 293T cell line. circKL, circular RNA KL, hsa_circ_0100312.

under specific pathogen-free conditions at $26^{\circ} \mathrm{C}$ and $20 \%$ humidity, with a 12-h light/dark cycle and ad libitum access to food and water. The tumors of the mice were measured with Vernier calipers every 4 days and their volumes calculated according to the following equation: $0.5 \mathrm{x}$ width ${ }^{2} \mathrm{x}$ length. After 28 days, the nude mice were euthanized by intraperitoneal injection of $80-100 \mu \mathrm{l}$ pentobarbital sodium $(100 \mathrm{mg} / \mathrm{kg})$. The tumors were then extracted and weighed.

A lung metastasis assay was also performed. SKNEP1 cells $\left(5 \times 10^{5}\right)$ were injected through the tail veins of nude mice (four mice per circKL group and vector group). After 8 weeks, lung tissues were excised while mice were anesthetized with sodium pentobarbital $(100 \mathrm{mg} / \mathrm{kg})$. Tumors were subsequently paraffin-embedded and cut into $4-\mu \mathrm{m}$ sections. The samples were dewaxed in xylene and rehydrated with an ethanol gradient. The sections were stained with hematoxylin (cat. no. ab245880; Abcam) for $5 \mathrm{~min}$ at room temperature and then with eosin (cat. no. ab245880; Abcam) for $2 \mathrm{~min}$ at room temperature. The number of macroscopically visible lung metastatic nodules was quantified and validated by a light microscope (magnification, x40, x100 and x200; Carl Zeiss AG).

Immunohistochemical staining. Tumor xenografts were fixed in $4 \%$ neutral formalin at room temperature for $24 \mathrm{~h}$. Histology sections ( $4 \mu \mathrm{m}$-thick) were prepared, deparaffinized using xylene and hydrated using a graded series of alcohols. The slides were incubated with Ki-67 antibodies (1:300 dilution; cat. no. 9449; Cell Signaling Technology, Inc.) overnight at $4^{\circ} \mathrm{C}$. Subsequently, horseradish-peroxidase-conjugated secondary antibodies (ready-to-use antibody 50-120 $\mu \mathrm{l}$; cat. no. 8125; Cell Signaling Technology, Inc.) were applied and samples were incubated for $45 \mathrm{~min}$ at room temperature. The resultant signal was visualized using 3,3'-diaminobenzidine color reagent staining at room temperature for $3 \mathrm{~min}$, after which the slides were counterstained with hematoxylin at room temperature for $5 \mathrm{~min}$ and dehydrated in ethanol and xylene. Finally, the staining was quantified and image acquisition was performed utilizing a light microscope (magnification, x200; Carl Zeiss AG).

Statistical analysis. All statistical analyses were performed using SPSS 23.0 software (SPSS, Inc.). Values are expressed as the mean \pm standard deviation of at least three independent experiments. Multigroup comparisons were performed using one-way ANOVA followed by Tukey's post hoc test. Comparisons between tumor and adjacent normal tissues were performed using a paired Student's t-test. A paired t-test was also used to compare the expression of circKL between two matched groups. $\mathrm{P}<0.05$ was considered to indicate a statistically significant difference.

\section{Results}

circKL has circular features and is downregulated in kidney cancer. Following a previous analysis of high-throughput microarray sequencing data by our group (26), RT-qPCR was performed in the present study to verify the expression level of the top five downregulated circRNAs in 10 pairs of kidney cancer samples and adjacent normal kidney samples (Fig. 1A). 
A

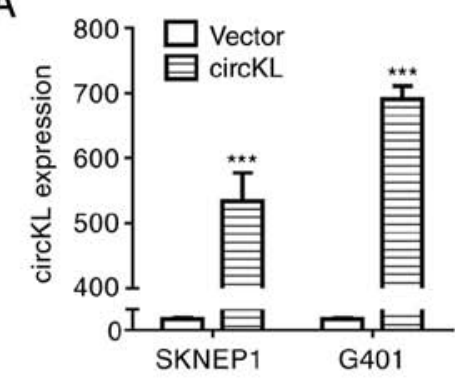

D

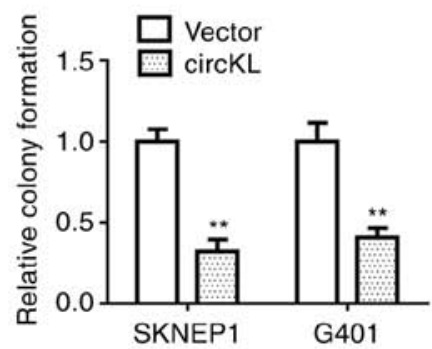

G

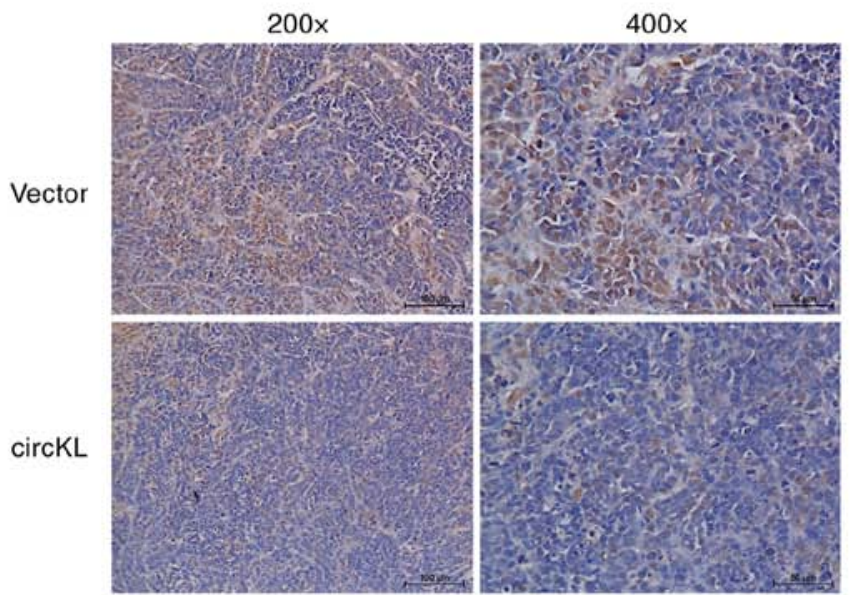

B

E
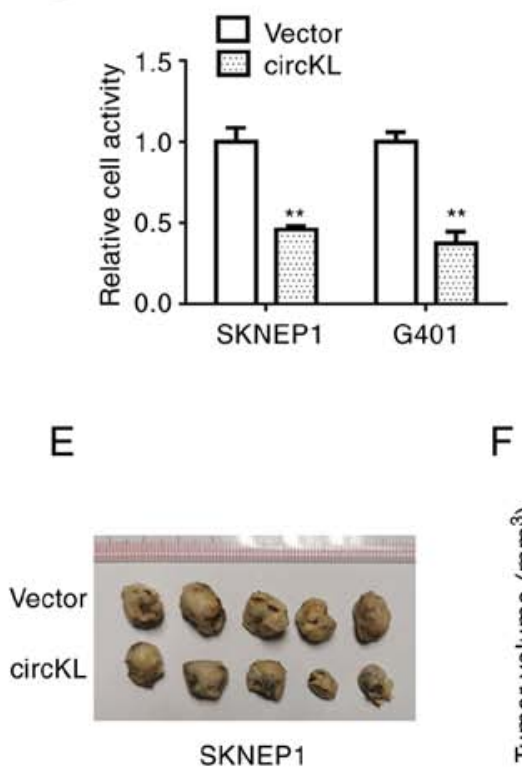

F
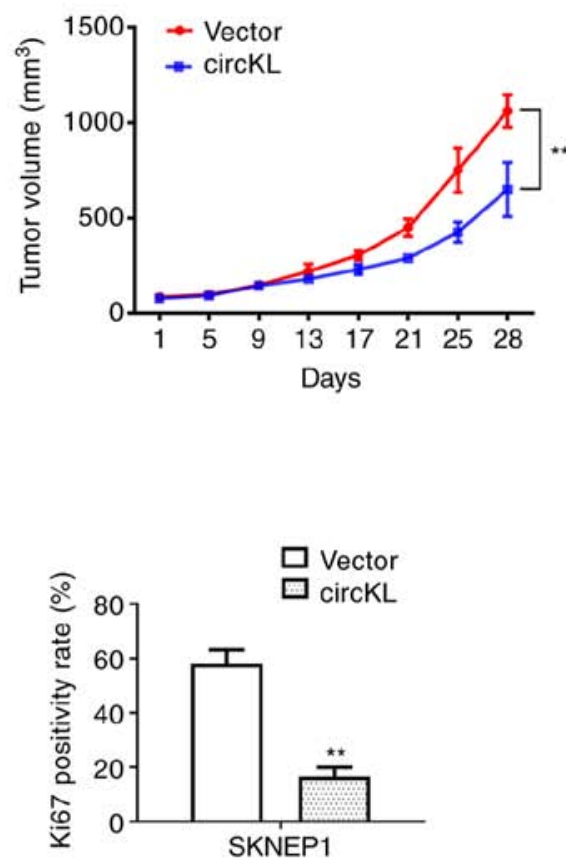

Figure 2. Overexpression of circKL inhibits the proliferation of kidney cancer cells. (A) The efficacy of overexpression of circKL in the SKNEP1 and G401 cell lines was assessed by reverse transcription-quantitative PCR. (B) Cell Counting Kit- 8 assays were used to evaluate cell proliferation of the SKNEP1 and G401 cell lines. (C and D) Colony-formation assays were performed with three different initial cell densities. (C) Representative images of the wells containing colonies in each group and (D) quantitative results of the colony-formation assay are provided. The cell assays were performed as three biological replicates. (E-G) Mouse xenograft models were established using the SKNEP1 kidney cancer cell line ( $\mathrm{n}=5$ for each group). (E) Images of tumors from each group at the end of the experiment. (F) The tumor volume in each group, which was determined every four days. (G) Immunohistochemical analysis of Ki-67 expression in xenograft tumors; representative images of are provided (scale bars, $50 \mu \mathrm{m}$ ) and the rate of Ki67 was quantitatively determined. ${ }^{* * *} \mathrm{P}<0.01,{ }^{* * * *} \mathrm{P}<0.001$ vs. vector. circKL, circular RNA KL, hsa_circ_0100312.

The results confirmed that circKL was significantly downregulated in the tumor parts of all tumor-normal tissue pairs. Furthermore, the expression levels of circKL were downregulated in kidney cancer cell lines compared with those in normal kidney 293 T cells (Fig. 1B). The circular structure and stability of circKL were further examined by performing RNase $\mathrm{R}$ and actinomycin $\mathrm{D}$ assays. The RNase $\mathrm{R}$ assay results revealed that, in contrast to linear KL mRNA, circKL was resistant to RNA exonuclease $(\mathrm{P}<0.01$; Fig. $1 \mathrm{C})$. Furthermore, actinomycin D assays confirmed that circKL had a significantly longer half-life than linear KL mRNA ( $\mathrm{P}<0.01$; Fig. 1D).

circKL overexpression inhibits the proliferation of kidney cancer cells. A vector that continuously expressed circKL to exogenously introduce circKL was constructed in the present study to explore the potential role of circKL in kidney cancer progression. G401 and SKNEP1 cells were transfected using a lentivirus that stably expressed circKL, after which the efficacy of the overexpression vector was verified $(\mathrm{P}<0.001$; Fig. 2A). CCK-8 and colony formation assays revealed that overexpression of circKL significantly suppressed the proliferation and colony-formation ability of G401 and SKNEP1 cell lines in vitro $(\mathrm{P}<0.01$; Fig. $2 \mathrm{~B}-\mathrm{D})$. Further examination of the anti-tumor function of circKL was performed in mouse xenograft assays. Tumor volume curves revealed that overexpression of circKL inhibited tumor growth. Similarly, in subcutaneous tumors, the maximum tumor diameter and mean volume in the circKL group $\left(0.984 \mathrm{~cm}\right.$ and $650.467 \mathrm{~mm}^{3}$, 


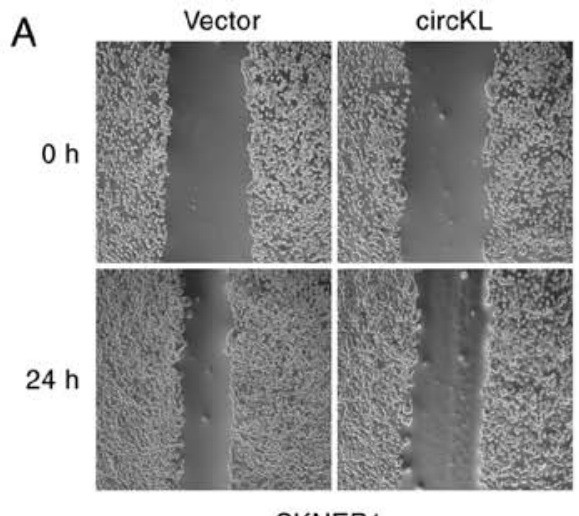

SKNEP1

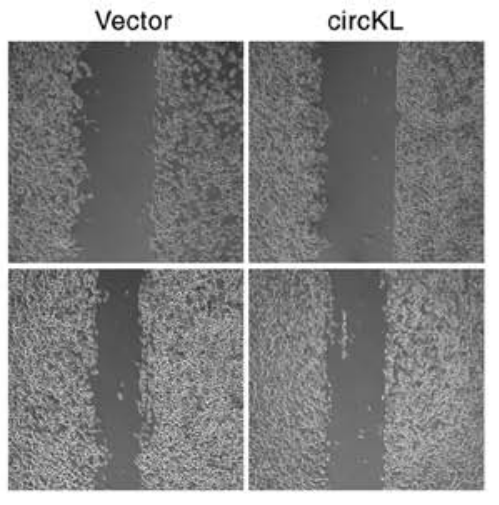

G401
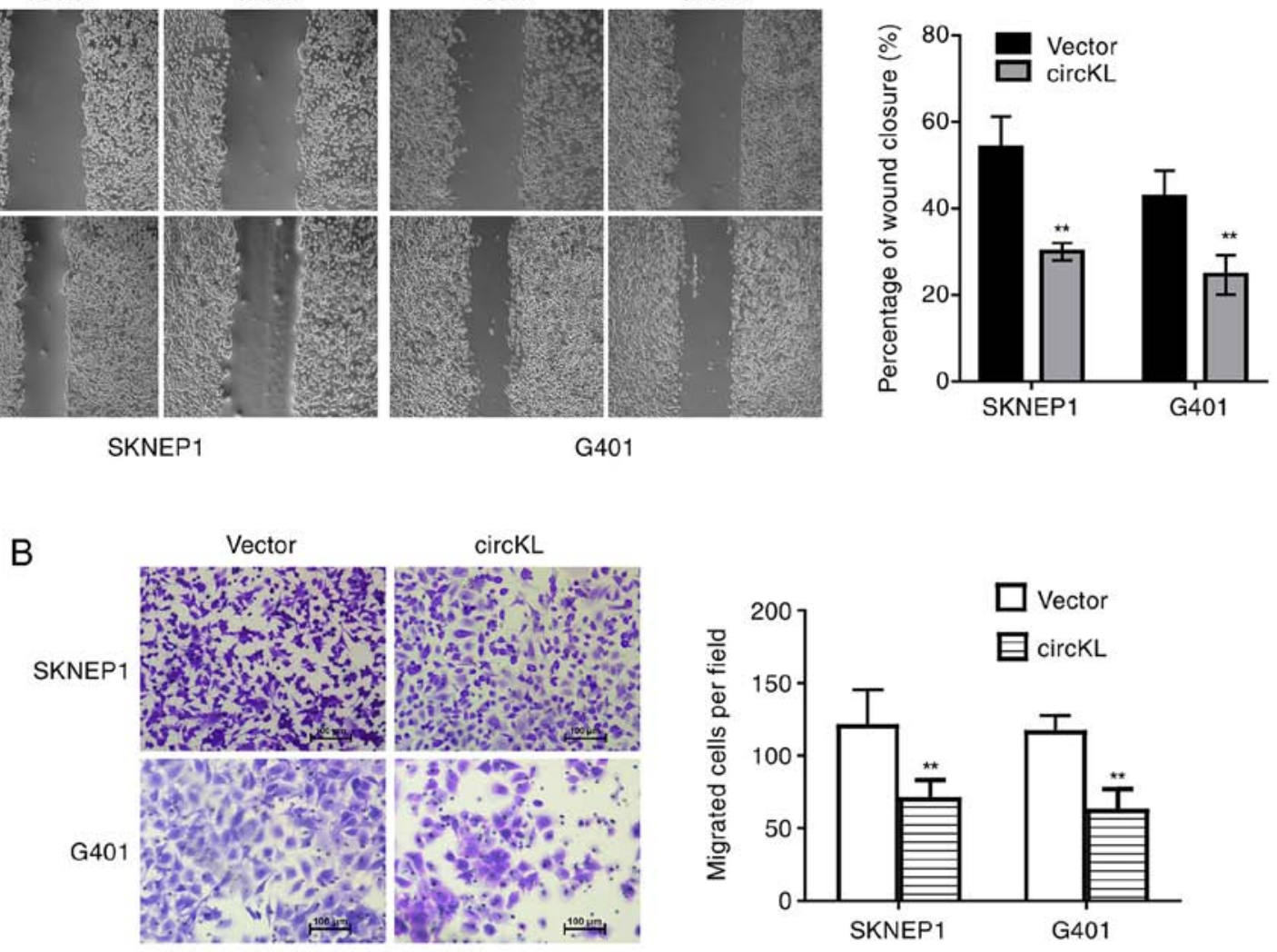

C
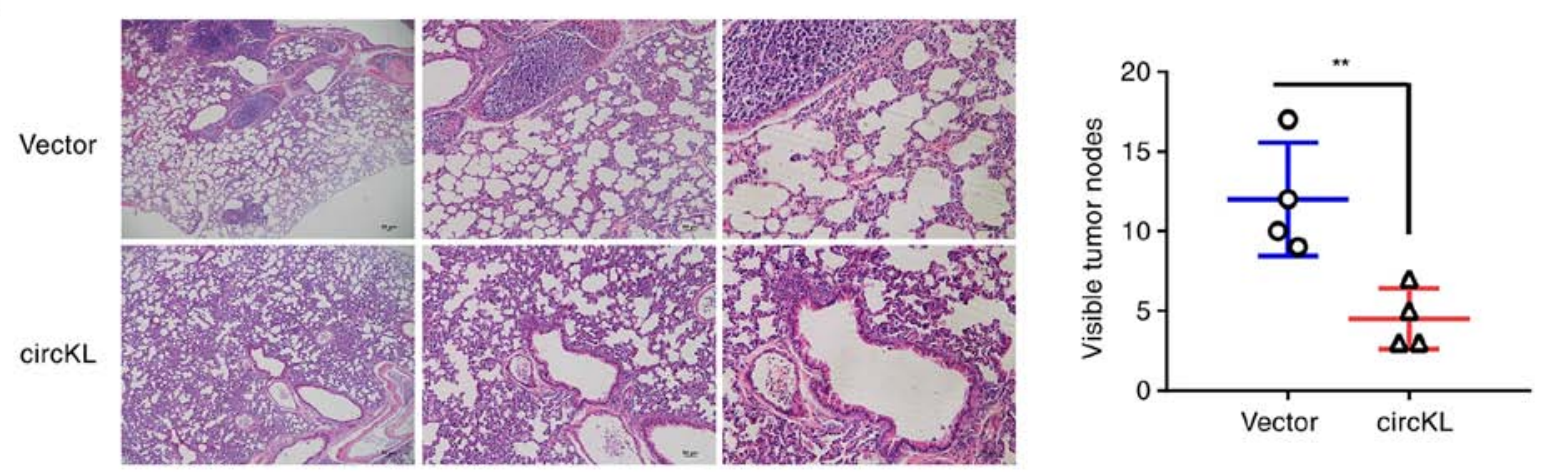

Figure 3. Overexpression of circKL inhibits metastasis of kidney cancer cells. (A) Wound-healing assay; images of scraped areas of in SKNEP1 and G401 cells at 0 and $24 \mathrm{~h}$ are presented and the percentage of wound closure was quantified. (B) A Transwell assay was performed to investigate the migration ability of SKNEP1 and G401 cells. Representative images of cells transgressed through the membrane are provided (scale bars, $100 \mu \mathrm{m}$ ) and the transgression rate was quantitatively determined. The cell experiments were performed as three biological replicates. (C) In the in vivo model, the number of lung metastases (SKNEP1 cell line) was determined. HE-stained sections of lung metastases were presented (scale bars, $50 \mu \mathrm{m}$ ). A total of four mice were used in each group. ${ }^{* *} \mathrm{P}<0.01$ vs. vector. circKL, circular RNA KL, hsa_circ_0100312.

respectively) were significantly smaller than those in the vector group (1.249 $\mathrm{cm}$ and $1061.87 \mathrm{~mm}^{3}$, respectively) after 28 days $(\mathrm{P}<0.01 ;$ Fig. $2 \mathrm{E}$ and $\mathrm{F})$. In addition, Ki67 protein expression in the murine xenograft tumors of the two groups was analyzed by immunohistochemistry. The results demonstrated that Ki67 expression was markedly decreased in the tumor tissues of the circKL overexpression vector group (Fig. 2G).

circKL overexpression inhibits metastasis of kidney cancer cells. Migration and invasion assays were performed to investigate whether circKL was able to suppress the metastatic capacity of kidney cancer cells. The results revealed that upregulation of circKL significantly inhibited the percentage of wound closure in G401 and SKNEP1 cells ( $\mathrm{P}<0.01$; Fig. 3A). The results of the Transwell assay demonstrated that circKL overexpression reduced the migration of G401 and SKNEP1 cells $(\mathrm{P}<0.01$; Fig. 3B). In concordance with the in vitro experimental results, circKL overexpression inhibited the metastasis of SKNEP1 cells in murine xenograft models in vivo $(\mathrm{P}<0.01$; Fig. $3 \mathrm{C})$.

circKL acts as a sponge of miR-182-5p in kidney cancer. After different cellular proportions were detected by qPCR, it was revealed that circKL predominantly existed in the cytoplasm of cells (Fig. 4A). The Circular RNA Interactome database (freely accessible at http://circinteractome.nia.nih.gov) was therefore used to evaluate the potential interactions between 
A

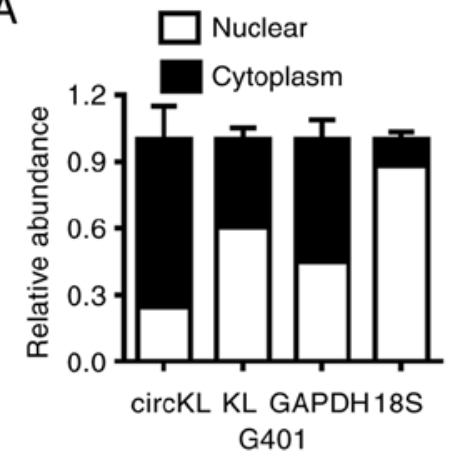

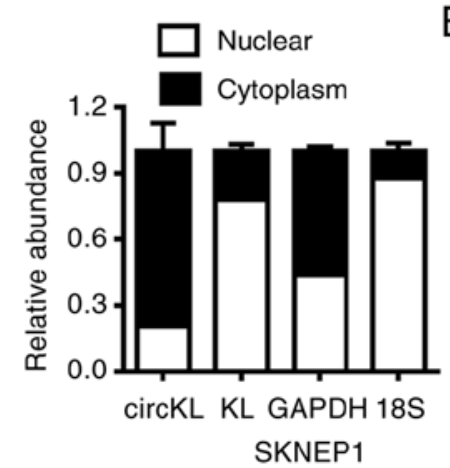

B

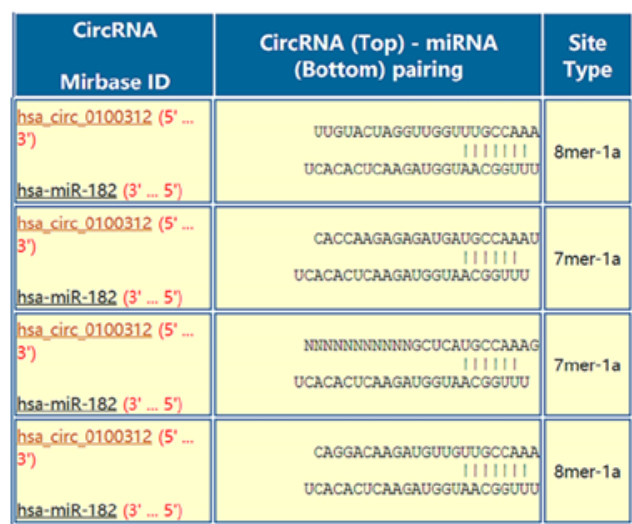

C

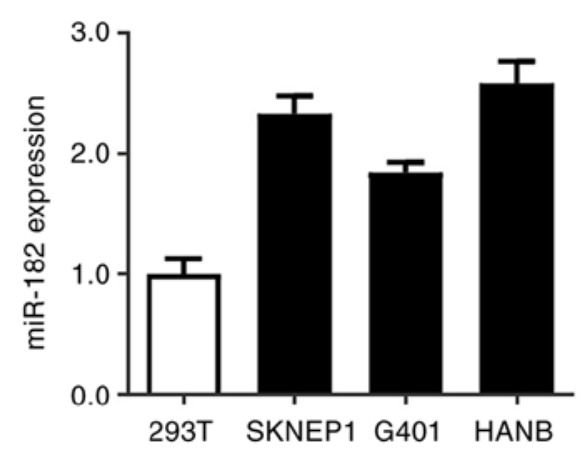

D MS2bs-Rluc

MS2bs-circKL

III MS2bs-circKL-mut

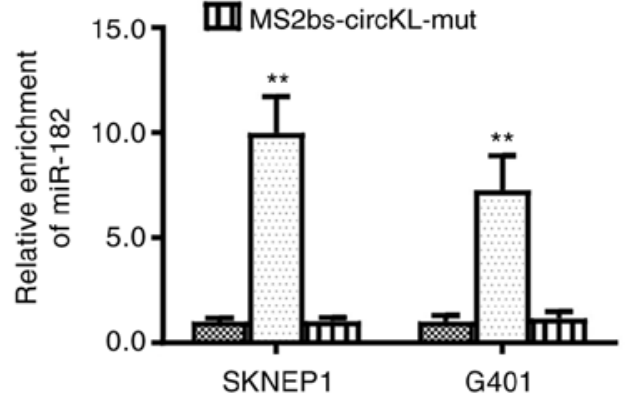

Figure 4. circKL is a sponge of miR-182 in kidney cancer. (A) 18S, GAPDH, circKL and KL expression in nuclear and cytoplasmic fractions were analyzed by reverse transcription-quantitative PCR. (B) Predicted binding sites of miR-182-5p within the circKL sequence. (C) Relative expression level of miR-182-5p in kidney cancer cell lines compared with that in 293T cells. (D) MS2-based radioimmunoprecipitation assay of cells transfected with MS2bs-circKL plasmid, MS2bs-circKL-mt plasmid or Rluc control plasmid. The cell assays were performed as three biological replicates. ${ }^{* *} \mathrm{P}<0.01$ vs. MS2bs-Rluc. RIP, RNA immunoprecipitation. Rluc, Renilla luciferase; circKL, circular RNA KL, hsa_circ_0100312; miR, microRNA; mut, mutant type.

circRNA and various miRNAs (27). Among the miRNA candidates, only miR-182-5p was predicted to bind to the circKL sequence at four possible interaction sites (Fig. 4B). In kidney cancer cell lines, RT-qPCR analysis revealed that miR-182-5p was significantly upregulated (Fig. 4C). Furthermore, the AGO2-related RIP assay confirmed the direct interaction between circKL and miR-182-5p. In addition, it was determined that miR-182-5p was predominantly enriched in the MS2bs-circKL overexpression vector group $(\mathrm{P}<0.01$; Fig. 4D), indicating that circKL directly interacted with miR-182-5p and may act as a sponge for miR-182-5p.

circKL inhibits kidney cancer progression through the circKL-miR-182-5p-FBXW7 axis. TargetScan (http://www. targetscan.org) was used to predict the potential targeting genes of miR-182-5p (27). Among the possible genes, FBXW7 was identified as a putative downstream target gene of miR-182-5p (Fig. 5A). The results of the qPCR analysis revealed that FBXW7 was markedly downregulated in kidney cancer cells (Fig. 5B). Whether miR-182-5p was able to directly bind to the 3'-UTR of FBXW7 mRNA was subsequently examined. The relative luciferase activity of G401 and SKNEP1 kidney cancer cells was significantly decreased following the transfection of miR-182-5p and the wild-type 3'-UTR-FBXW7 plasmids. However, after co-transfection with the mutated luciferase reporter vector, no such effect was observed ( $\mathrm{P}<0.01$; Fig. $5 \mathrm{C})$. The exogenous introduction of miR-182-5p contributed to the reduction of FBXW7 mRNA expression levels $(\mathrm{P}<0.01$; Fig. 5D). In addition, AGO2-related RIP assays revealed that circKL, miR-182-5p and FBXW7 were all highly enriched in the anti-AGO2 G401 and SKNEP1 kidney cancer cell groups $(\mathrm{P}<0.01$; Fig. 5E). Furthermore, the mRNA level of FBXW7 was markedly decreased following circKL overexpression $(\mathrm{P}<0.01$; Fig. 5F). After transfection with miR-182-5p mimics, FBXW7 protein was decreased in both G401 and SKNEP1 kidney cancer cell lines (Fig. 5G). Western blot analysis also revealed that circKL overexpression significantly increased FBXW7 protein levels (Fig. 5H).

\section{Discussion}

circRNAs have become a focus of ncRNA research in recent years. Due to their high expression efficiency, structural stability and disease specificity, scientists have been able to utilize high-throughput sequencing technology and bioinformatics analysis to discover and study various circRNAs (28). circRNAs are novel ncRNAs that occur as covalently closed loops. They are widely expressed in mammalian tissues and exhibit tissue-specific and cell-specific expression patterns (29). Although originally thought to be useless products of mRNA pre-splicing, these unique ncRNAs with circular structures are currently recognized as relatively well-established biomarkers in cancer diagnosis (30). With the popularization of high-throughput technology, hundreds of circRNAs have 
A

\begin{tabular}{|c|c|c|}
\hline & $\begin{array}{l}\text { Predicted consequential pairing of target region (top) } \\
\text { and miRNA (bottom) }\end{array}$ & $\begin{array}{l}\text { Site } \\
\text { type }\end{array}$ \\
\hline $\begin{array}{l}\text { Position 555-561 of FBXW7 3' UTR } \\
\text { hsa-miR-182-5p }\end{array}$ & 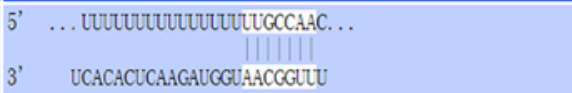 & $\begin{array}{c}7 \mathrm{mer}- \\
\mathrm{m} 8\end{array}$ \\
\hline $\begin{array}{l}\text { Position } 565-571 \text { of FBXW7 3' UTR } \\
\text { hsa-miR-182-5p }\end{array}$ & $\begin{array}{l}5, \quad \text {. . UUUTUUUGGCCAACCALUGCCAAU... } \\
\text { 3, } \quad \text { UCACACUCAAGAUGGUAACGGULU }\end{array}$ & $\begin{array}{c}7 \mathrm{mer}- \\
\mathrm{m} 8\end{array}$ \\
\hline
\end{tabular}

C

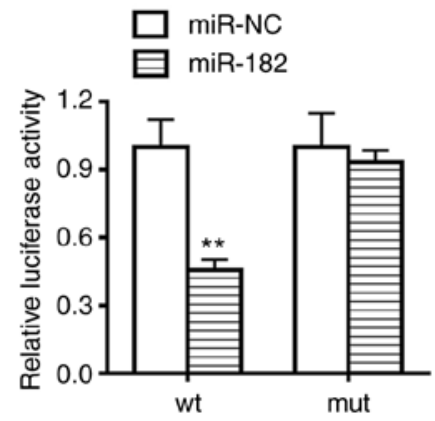

E

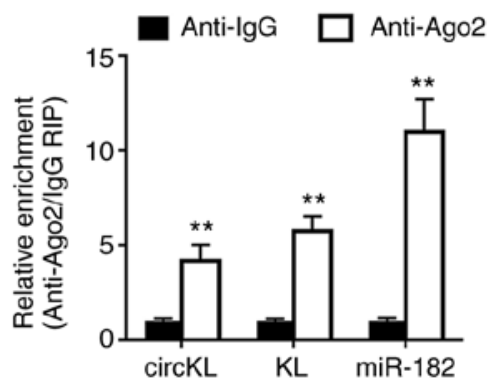

G
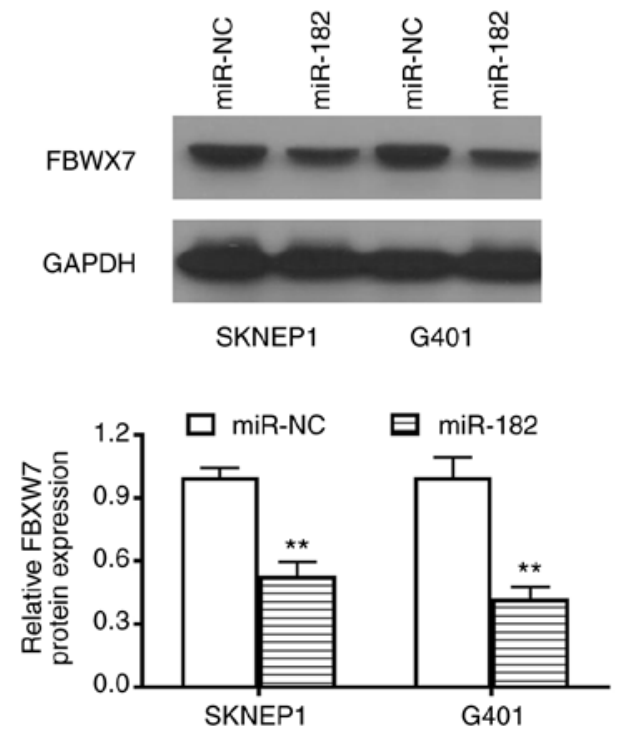

$\mathrm{F}$

$\mathrm{H}$
$\mathrm{B}$

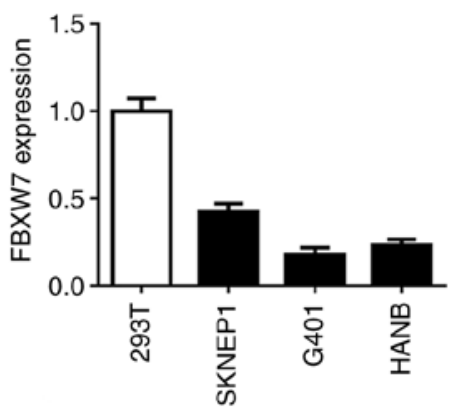

D

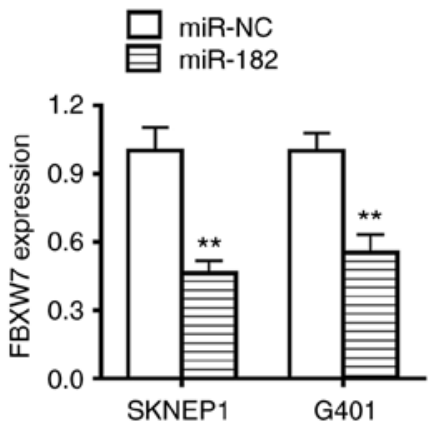

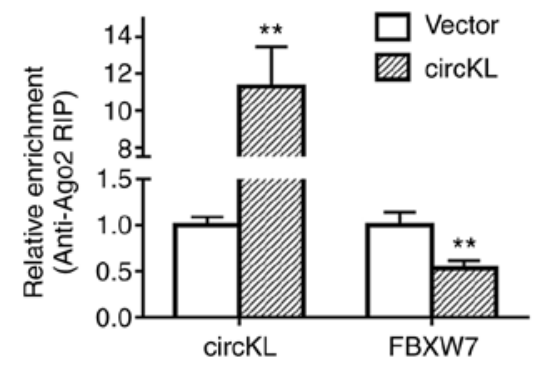

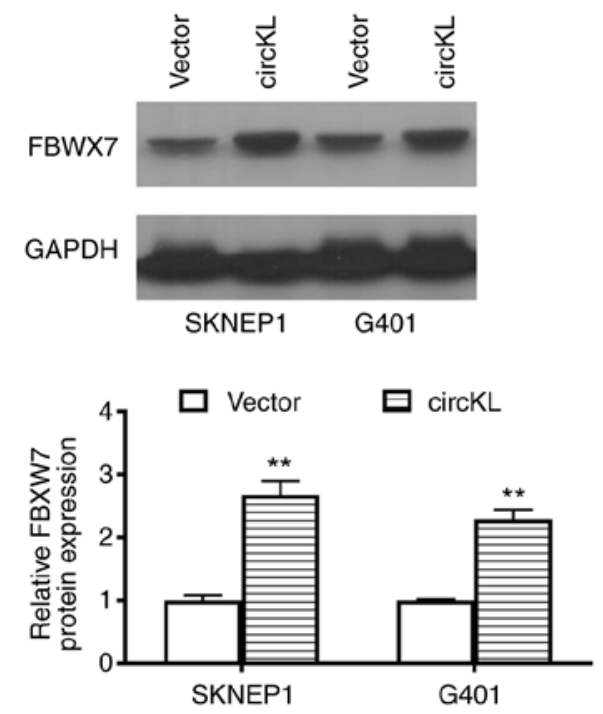

Figure 5. circKL inhibits kidney cancer progression through the circKL-miR-182-5p-FBXW7 axis. (A) Two sequences from the 3'UTR of FBXW7 were predicted as a downstream target of miR-182-5p, according to the TargetScan online website. (B) Relative expression level of FBXW7 in kidney cancer cell lines. (C) Luciferase reporter assay using SKNEP1 and G401 cell lines co-transfected with miR-182-5p mimics and luciferase reporter plasmid containing the wild/mutant-type fragment from the 3'-UTR of FBXW7. (D) Overexpression of miR-182-5p contributed to the reduction of FBXW7 expression in SKNEP1 and G401 cell lines, as detected by reverse transcription-quantitative PCR analysis. (E) Enrichment of circKL, FBXW7 and miR-182-5p on AGO2 assessed by RIP assay. (F) Enrichment of FBXW7 to AGO2 was decreased after overexpression of circKL. (G) Overexpression of miR-182-5p contributed to the reduction of FBXW7 expression in SKNEP1 and G401 cell lines, as detected by western blot analysis. (H) Overexpression of circKL increased the expression of FBXW7 in SKNEP1 and G401 cell lines, as detected by western blot analysis. Each assay was performed as three biological replicates. ${ }^{* *} \mathrm{P}<0.01 \mathrm{vs}$. miR-NC, vector. FBXW7, F-box and WD-40 domain protein 7; wt, wild-type; mut, mutant-type; NC, negative control; Ago2, argonaute RISC catalytic component 2; RIP, RNA immunoprecipitation; circKL, circular RNA KL, hsa_circ_0100312; miR, microRNA. 
been discovered as novel predictive biomarkers and promising therapeutic targets for cancer therapy in recent years (31). For instance, circAGO2 was indicated to harbor oncogenic properties through activating human antigen $\mathrm{R}$ in different types of cancer (32). Furthermore, circAHNAK1 (Desmoyokin) and circRNA of Homo sapiens $\mathrm{G}$ protein subunit $\beta 1$ have been identified as critical regulatory factors for competing endogenous (ce)RNA mechanisms in triple-negative breast cancer $(33,34)$. Certain circRNAs, including circfam114a2 (35) and circitch (36), have been determined to act as tumor suppressors through different molecular mechanisms. However, the potential molecular mechanisms and biological roles of circRNAs in kidney cancer have remained largely elusive.

For the present study, high-throughput circRNA microarray data from a previous study by our group were analyzed to screen for the differentially expressed circRNAs in three pairs of kidney cancer tissues (26). circKL was identified as a significantly downregulated circRNA in both kidney cancer cells and tissues. A circKL overexpression plasmid was then constructed to investigate the function of circKL in kidney cancer. circKL overexpression significantly inhibited the proliferation and migration of kidney cancer cells in vitro and in vivo. RIP analysis and a luciferase reporter assay were also performed in the present study to reveal the underlying mechanisms of the actions of circKL. The results demonstrated that circKL inhibited the progression of kidney cancer via miR-182-5p sponging, which upregulated FBXW7 expression.

circRNAs have been known to serve as miRNA sponges for several years (37). Theoretically, ceRNAs, mRNAs, long non-coding RNAs and circRNAs are able to regulate and communicate through the competitive binding of shared miRNAs (38). In the present study, miR-182-5p was indicated to interact with circKL in kidney cancer. miR-182-5p promotes the progression of hepatocellular carcinoma by inhibiting forkhead box (FOX)O3a expression, which is a potential predictor of early hepatocellular carcinoma recurrence in patients who underwent curative surgery (39). Regulated by circRNA_0025202, miR-182-5p attenuates tamoxifen resistance by downregulating FOXO3a expression in breast cancer (40). In addition, circRNA BCRC-3 suppresses cancer cell metastasis and proliferation through the miR-182-5p/p27 axis in bladder cancer (41). FBXW7 encodes a member of the F-box protein family, which is a motif characterized by $\sim 40$ amino acids that was originally identified in the cell cycle. The F-box protein has an important role in phosphorylation-dependent ubiquitination and is one of the four subunits of the ubiquitin protein ligase complex, Skp1-Cullin-F-box. FBXW7 has been proven to be an important tumor suppressor in multiple types of cancer $(42,43)$. Of note, FBXW7 expression may be regulated by its circular transcription (44). CircFBXW7 inhibits the proliferation and invasion of glioma and colorectal cancer cells by translating a $21 \mathrm{kDa}$ novel protein (FBXW7-185AA) and sponging miRNA $(44,45)$. In the present study, FBXW7 was significantly upregulated following circKL overexpression in kidney cancer cells, which was consistent with the results of previous studies (43). These findings identified the important roles of circRNAs in the downstream regulation and modulation of cancer progression.

In summary, the present study elucidated the biological role of circKL in the growth and metastasis of kidney cancer through the miR-182-5p/FBXW7 axis. The results of the current study are of great significance for the development of novel treatment strategies and potential prognostic biomarkers for patients with kidney cancer.

\section{Acknowledgements}

Not applicable.

\section{Funding}

This study was supported by grants from the Clinical Research Project of Shenzhen Healthcare Research Project (grant no. SZLY2018015), Shenzhen Fund for Guangdong Provincial High-level Clinical Key Specialties (grant no. SZGSP012) and Shenzhen Key Medical Discipline Construction Fund (grant no. SZXK034).

\section{Availability of data and materials}

The datasets used and analyzed during the current study are available from the corresponding author on reasonable request.

\section{Authors' contributions}

JC performed data analyses and wrote the initial manuscript. JC and SL designed the study and revised the manuscript. JC, UY, LL, SC and HX performed the cell and animal experiments. $\mathrm{XY}$ performed the bioinformatics analysis. MY contributed clinical information and samples, as well as technical support for multiple software applications. JC and SL checked and confirmed the authenticity of the raw data. All authors read and approved the final manuscript.

\section{Ethics approval and consent to participate}

All experimental protocols were approved by the Ethics Committee of Shenzhen Children's Hospital (Shenzhen, China; no. 201903902). The legal guardians of all participants provided written informed consent. All experiments involving animals were approved by the Animal Ethics Committee of Shenzhen Children's Hospital (Shenzhen, China; no. 20200102).

\section{Patient consent for publication}

Not applicable.

\section{Competing interests}

The authors declare that they have no competing interests.

\section{References}

1. Treger TD, Chowdhury T, Pritchard-Jones K and Behjati S: The genetic changes of Wilms tumour. Nat Rev Nephrol 15: 240-251, 2019.

2. Chowdhury N and Drake CG: Kidney cancer: An overview of current therapeutic approaches. Urol Clin North Am 47: 419-431, 2020.

3. Anvar Z, Acurzio B, Roma J, Cerrato F and Verde G: Origins of DNA methylation defects in Wilms tumors. Cancer Lett 457: $119-128,2019$. 
4. Lange J, Peterson SM, Takashima JR, Grigoriev Y, Ritchey ML Shamberger RC, Beckwith JB, Perlman E, Green DM and Breslow NE: Risk factors for end stage renal disease in non-WT1-syndromic Wilms tumor. J Urol 186: 378-386, 2011.

5. Dome JS, Graf N, Geller JI, Fernandez CV, Mullen EA, Spreafico F, Van den Heuvel-Eibrink M and Pritchard-Jones K: Advances in Wilms tumor treatment and biology: Progress through international collaboration. J Clin Oncol 33: 2999-3007, 2015.

6. Clericuzio CL and Johnson C: Screening for Wilms tumor in high-risk individuals. Hematol Oncol Clin North Am 9: 1253-1265, 1995 .

7. Goodall GJ and Wickramasinghe VO: RNA in cancer. Nat Rev Cancer 21: 22-36, 2021.

8. Qu S, Yang X, Li X, Wang J, Gao Y, Shang R, Sun W, Dou K and Li H: Circular RNA: A new star of noncoding RNAs. Cancer Lett 365: 141-148, 2015.

9. Ebbesen KK, Hansen TB and Kjems J: Insights into circular RNA biology. RNA Biol 14: 1035-1045, 2017.

10. Chen LL: The expanding regulatory mechanisms and cellular functions of circular RNÂs. Nat Rev Mol Cell Biol 21: 475-490, 2020.

11. Vo JN, Cieslik M, Zhang Y, Shukla S, Xiao L, Zhang Y, Wu YM, Dhanasekaran SM, Engelke CG, Cao X, et al: The landscape of circular RNA in cancer. Cell 176: 869-881.e13, 2019.

12. Han B, Chao J and Yao H: Circular RNA and its mechanisms in disease: From the bench to the clinic. Pharmacol Ther 187: 31-44, 2018.

13. Su Y, Lv X, Yin W, Zhou L, Hu Y, Zhou A and Qi F: circRNA Cdrlas functions as a competitive endogenous RNA to promote hepatocellular carcinoma progression. Aging (Albany NY) 11: 8182-8203, 2019

14. Yang W, Yang $X$, Wang X, Gu J, Zhou D, Wang Y, Yin B, Guo J and Zhou M: Silencing CDRlas enhances the sensitivity of breast cancer cells to drug resistance by acting as a miR-7 sponge to down-regulate REG $\gamma$. J Cell Mol Med 23: 4921-4932, 2019.

15. Zou Y, Zheng S, Deng X, Yang A, Xie X, Tang H and Xie X: The role of circular RNA CDR1as/ciRS-7 in regulating tumor microenvironment: A pan-cancer analysis. Biomolecules 9: 429, 2019.

16. Memczak S, Jens M, Elefsinioti A, Torti F, Krueger J, Rybak A, Maier L, Mackowiak SD, Gregersen LH, Munschauer M, et al: Circular RNAs are a large class of animal RNAs with regulatory potency. Nature 495: 333-338, 2013

17. Zou Y, Zheng S, Deng X, Yang A, Kong Y, Kohansal M, Hu X and Xie X: Diagnostic and prognostic value of circular RNA CDR1as/ciRS-7 for solid tumours: A systematic review and meta-analysis. J Cell Mol Med 24: 9507-9517, 2020.

18. Jiang C, Zeng X, Shan R, Wen W, Li J, Tan J, Li L and Wan R The emerging picture of the roles of circRNA-CDRlas in cancer. Front Cell Dev Biol 8: 590478, 2020.

19. Wang J, Zhao X, Wang Y, Ren F, Sun D, Yan Y, Kong X, Bu J, Liu M and Xu S: circRNA-002178 act as a ceRNA to promote PDL1/PD1 expression in lung adenocarcinoma. Cell Death Dis 11: 32,2020

20. Dai X, Zhang N, Cheng Y, Yang T, Chen Y, Liu Z, Wang Z, Yang $\mathrm{C}$ and Jiang Y: RNA-binding protein trinucleotide repeat-containing $6 \mathrm{~A}$ regulates the formation of circular RNA circ0006916, with important functions in lung cancer cells. Carcinogenesis 39: 981-992, 2018

21. Lin G, Wang S, Zhang X and Wang D: Circular RNA circPLK1 promotes breast cancer cell proliferation, migration and invasion by regulating miR-4500/IGF1 axis. Cancer Cell Int 20: 593 , 2020.

22. Kong Y, Yang L, Wei W, Lyu N, Zou Y, Gao G, Ou X, Xie X and Tang H: CircPLK1 sponges miR-296-5p to facilitate triple-negative breast cancer progression. Epigenomics 11: 1163-1176, 2019.

23. Zou Y, Zheng S, Xiao W, Xie X, Yang A, Gao G, Xiong Z, Xue Z, Tang $\mathrm{H}$ and Xie X: circRAD18 sponges miR-208a/3164 to promote triple-negative breast cancer progression through regulating IGF1 and FGF2 expression. Carcinogenesis 40: 1469-1479, 2019.

24. Huang Y, Zhang W, Song H and Sun N: A nomogram for prediction of distant metastasis in children with Wilms tumor: A study based on SEER database. J Pediatr Urol 16: 473.e1-473.e9, 2020
25. Chi J, Liu S, Wu Z, Shi Y, Shi C, Zhang T, Xiong B, Zeng Y and Dong $\mathrm{X}$ : circNSUN2 promotes the malignant biological behavior of colorectal cancer cells via the miR-181a-5p/ROCK2 axis. Oncol Rep 46: 142, 2021.

26. Cao J, Huang Z, Ou S, Wen F, Yang G, Miao Q, Zhang H, Wang Y, He X, Shan Y, et al: circ0093740 promotes tumor growth and metastasis by sponging miR-136/145 and upregulating DNMT3A in Wilms tumor. Front Oncol 11: 647352, 2021.

27. Dudekula DB, Panda AC, Grammatikakis I, De S, Abdelmohsen K and Gorospe M: CircInteractome: A web tool for exploring circular RNAs and their interacting proteins and microRNAs. RNA Biol 13: 34-42, 2016.

28. Jeck WR and Sharpless NE: Detecting and characterizing circular RNAs. Nat Biotechnol 32: 453-461, 2014.

29. Dong Y, He D, Peng Z, Peng W, Shi W, Wang J, Li B, Zhang C and Duan C: Circular RNAs in cancer: An emerging key player. J Hematol Oncol 10: 2, 2017.

30. Bolha L, Ravnik-Glavač M and Glavač D: Circular RNAs: Biogenesis, function, and a role as possible cancer biomarkers. Int J Genomic 2017: 6218353, 2017.

31. Li S and Han L: Circular RNAs as promising biomarkers in cancer: Detection, function, and beyond. Genome Med 11: 15, 2019.

32. Chen Y, Yang F, Fang E, Xiao W, Mei H, Li H, Li D, Song H, Wang J, Hong M, et al: Circular RNA circAGO2 drives cancer progression through facilitating HuR-repressed functions of AGO2-miRNA complexes. Cell Death Differ 26: 1346-1364, 2019.

33. Xiao W, Zheng S, Zou Y, Yang A, Xie X, Tang H and Xie X: CircAHNAK 1 inhibits proliferation and metastasis of triple-negative breast cancer by modulating miR-421 and RASA1. Aging (Albany NY) 11: 12043-12056, 2019.

34. Liu P, Zou Y, Li X, Yang A, Ye F, Zhang J, Wei W and Kong Y: circGNB1 facilitates triple-negative breast cancer progression by regulating miR-141-5p-IGF1R axis. Front Genet 11: 193, 2020.

35. Liu T, Lu Q, Liu J, Xie S, Feng B, Zhu W, Liu M, Liu Y, Zhou X, Sun W, et al: Circular RNA FAM114A2 suppresses progression of bladder cancer via regulating $\triangle$ NP63 by sponging miR-762. Cell Death Dis 11: 47, 2020

36. Li Y, Ge YZ, Xu L and Jia R: Circular RNA ITCH: A novel tumor suppressor in multiple cancers. Life Sci 254: 117176, 2020

37. Verduci L, Strano S, Yarden Y and Blandino G: The circRNA-microRNA code: Emerging implications for cancer diagnosis and treatment. Mol Oncol 13: 669-680, 2019.

38. Tay Y, Rinn J and Pandolfi PP: The multilayered complexity of ceRNA crosstalk and competition. Nature 505: 344-352, 2014.

39. Cao MQ, You AB, Zhu XD, Zhang W, Zhang YY, Zhang SZ, Zhang KW, Cai H, Shi WK, Li XL, et al: miR-182-5p promotes hepatocellular carcinoma progression by repressing FOXO3a. J Hematol Oncol 11: 12, 2018.

40. Sang Y, Chen B, Song X, Li Y, Liang Y, Han D, Zhang N, Zhang H, Liu Y, Chen T, et al: circRNA_0025202 regulates tamoxifen sensitivity and tumor progression via regulating the miR-182-5p/FOXO3a axis in breast cancer. Mol Ther 27: $1638-1652,2019$

41. Xie F, Li Y, Wang M, Huang C, Tao D, Zheng F, Zhang H, Zeng F, Xiao X and Jiang G: Circular RNA BCRC-3 suppresses bladder cancer proliferation through $\mathrm{miR}-182-5 \mathrm{p} / \mathrm{p} 27$ axis. Mol Cancer 17: 144, 2018.

42. Yeh $\mathrm{CH}$, Bellon $\mathrm{M}$ and Nicot C: FBXW7: A critical tumor suppressor of human cancers. Mol Cancer 17: 115, 2018.

43. Yumimoto K and Nakayama KI: Recent insight into the role of FBXW7 as a tumor suppressor. Semin Cancer Biol 67:1-15, 2020.

44. Yang Y, Gao X, Zhang M, Yan S, Sun C, Xiao F, Huang N, Yang X, Zhao K, Zhou H, et al: Novel role of FBXW7 circular RNA in repressing glioma tumorigenesis. J Natl Cancer Inst 110: 304-315, 2018

45. Xu Y, Qiu A, Peng F, Tan X, Wang J and Gong X: Exosomal transfer of circular RNA FBXW7 ameliorates the chemoresistance to oxaliplatin in colorectal cancer by sponging miR-18b-5p. Neoplasma 68: 108-118, 2021.

This work is licensed under a Creative Commons Attribution-NonCommercial-NoDerivatives 4.0 International (CC BY-NC-ND 4.0) License. 